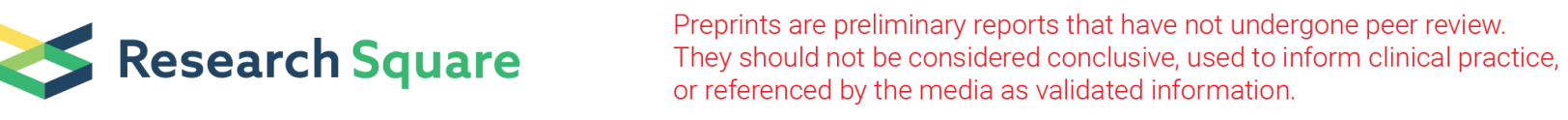

\title{
Knockdown long noncoding RNA SLC8A1-AS1 attenuate cell invasion and migration in glioma via suppression of Wnt- $\beta$ catenin signaling pathways
}

Ling He

The first affiliated hospital of wannan medical college https://orcid.org/0000-0003-0260-1822

Hui Yang

The first affiliated hospital of wannan medical college

Xiao-long Zhu

The first affiliated hospital of wannan medical college

Yan Zhang

The first affiliated hospital of wannan medical college

Kun Lv ( $\nabla$ lvkun315@126.com )

Research

Keywords: IncRNA SLC8A1-AS1; glioma; Wnt/ß-catenin; EMT

Posted Date: May 22nd, 2020

DOI: https://doi.org/10.21203/rs.3.rs-30035/v1

License: (1) (i) This work is licensed under a Creative Commons Attribution 4.0 International License.

Read Full License 


\section{Abstract}

Background: Glioma, as the most common aggressive malignant tumor in the central nervous system, is still an insurmountable disease in neural system. The potential mechanism of its carcinogenesis remains largely unclear.

Methods: In the present study, we identified dysregulated IncRNA solute carrier family 8 member A1 antisense RNA 1 (SLC8A1-AS1) as associated with glioma based on The Cancer Genome Atlas $\triangle T C G A \rrbracket$ data. Validation experiment was conducted to confirm a high expression level of IncRNA SLC8A1-AS1 in glioma tissues.

Results: Down-regulation of IncRNA SLC8A1-AS1 suppressed proliferation, clone formation, migration and invasion of glioma cells in vitro and in vivo. Moreover, IncRNA SLC8A1-AS1 silencing decreased the activity of the Wnt/ $\beta$-catenin pathway and suppressed the epithelial to mesenchymal transition (EMT) in glioma cells.

Conclusions: Collectively, these findings provide a novel insights into the function and mechanism of IncRNA SLC8A1-AS1 in the pathogenesis of glioma and highlight its potential as a therapeutic target for glioma intervention.

\section{Background}

Glioma is the most common and aggressive tumor in the nervous system, and accounts for about $80 \%$ of primary malignant brain tumors[1,2]. Although there have been advances in glioma therapy in the past decades, overall survival of most patients with glioma have not changed significantly, for which the median survival time is only approximately 14 months[3]. Therefore, it is an urgent need to clarify molecular mechanisms and identify new therapeutic targets for glioma.

Recent integrative genomic studies have revealed that $98 \%$ of the human genome transcripts are noncoding RNA (ncRNA) with limited or no protein-coding capacity [4]. Long non-coding RNAs (IncRNAs), transcripts $>200$ nucleotides in length are important new members of the ncRNA family[5]. The dysregulated IncRNAs expression has been implicated in glioma[6,7], however, most latent functions of these IncRNAs are still unknown.

In the present study, a high expression level of IncRNA solute carrier family 8 member A1 antisense RNA 1 (SLC8A1-AS1) was found in glioma tissues by analysis of the gene-expression dataset of TCGA. We assessed the expression of IncRNA SLC8A1-AS1 in 67 glioma samples and 7 histologically normal tissues, and confirmed the up-regulation of IncRNA SLC8A1-AS1 in glioma. Functional studies were further performed and the results suggested that knockdown of IncRNA SLC8A1-AS1 could result in the inhibition of cell proliferation, clone formation, invasion and migration in vitro and tumor growth in vivo. More over, we demonstrated that IncRNA SLC8A1-AS1 is involved in glioma cells progression by activating of Wnt/ $\beta$-catenin signaling pathway. 


\section{Materials And Methods}

\section{Tissue samples}

In this study, tumor tissues were obtained from 67 glioma patients, and non-neoplastic brain samples were obtained from 7 traumatic brain injury patients at the Department of Neurosurgery of The first affiliated Hospital of Wannan Medical College from February 2014 to October 2017. Two pathologists evaluated all specimens according to the World Health Organization (WHO) guidelines. No local or systemic treatments were administered to these patients before surgery. The tissues were immediately frozen in liquid nitrogen and stored at $-80^{\circ} \mathrm{C}$ until use. All of the patients provided signed, informed consent before the use of these clinical materials for research purposes. The use of these archival tissues in this study was approved by the Ethics Committee of The first affiliated Hospital of Wannan Medical College.

\section{Cell culture}

Human U87MG and LN382 glioblastoma cell lines were obtained from ATCC (American Type Culture Collection, USA). All cell lines were routinely cultured at $37^{\circ} \mathrm{C}$ in a $5 \% \mathrm{CO}_{2}$ humidified atmosphere in Dulbecco's-modified Eagle medium (DMEM) with 10\% fetal bovine serum (FBS, Gibco).

\section{Real-time RT-PCR}

Total RNA was extracted from the transfected cells with TRIzol (Invitrogen) and 0.4 $\mu$ g RNA was used to synthesize cDNA using a first strand cDNA synthesis kit (Termo scientifc, USA). The RNA concentration was examined using a NanoDrop 2000 spectrophotometer (Termoscientifc, USA). Real-time RT-PCR analysis was performed using the CFX-96 (BioRad, USA) according to the manufacturer's instructions. Data were normalized according to the level of GAPDH expression in each sample. GAPDH was used as

an internal control, and $2^{-} \triangle \triangle \mathrm{Ct}$ values were used to assess the relative expression of the target gene. The primers of GAPDH were synthesized by RiBoBio (Guangzhou, China)

qRT-PCR primers for IncRNA SLC8A1-AS1 were as follows:

Forward 5'-3': CAGTCGTGTTCGTCGCACTT

Reverse 5'-3': GCTGCCCGTGACGTTACCTAT

\section{Colony formation assay}

For the colony formation assay, the cells were plated in 6 -well plates at $2 \times 10^{2}$ cells per well and maintained in DMEM containing 10\% FBS for 2 weeks. After 2 weeks, the cells were washed two times with PBS, fixed with methanol and stained with rystal violet at the end of the time course prior to the capture of the representative images via camera. The number of colonies was counted under a microscope. All experiments were performed in triplicate. 


\section{Cell cycle assay}

Cell cycle analysis was performed by determining the DNA content with propidium iodide (PI) staining (BD Biosciences; San Jose, CA, USA). Briefly, U87MG and LN382 glioma cells were harvested, resuspended and stained with propidium iodide (PI; BD Biosciences) in the presence of RNase A for 20 min. Cells were analysed using a flow cytometer (BD Biosciences) according to the manufacturer's instructions.

\section{Wound healing assay}

Culture and transfection conditions for U87MG and LN382 cells were optimized to ensure a homogeneous and viable cell monolayer prior to wounding. One day before transfection, equal numbers of U87MG and LN382 cells $\left(5.0 \times 10^{5}\right)$ were seeded into 6-well tissue culture plates without antibiotics. Cells were then transfected with Noncontrol (100nM) and SLC8A1-AS1 siRNA (100 nM) using riboFECT ${ }^{\text {TM }}$ CP RiBoBio (Guangzhou, China), respectively. When the cell confluence reached about $90 \%$ at $24 \mathrm{~h}$ posttransfection, an artificial homogenous wound was created onto the monolayer with a sterile plastic $200 \mu \mathrm{L}$ micropipette tip. After wounding, the debris was removed by washing the cells with serum-free medium. Migration of cells into the wound was observed at 0 and $24 \mathrm{~h}$, respectively. Cells that migrated into the wounded area or cells with extended protrusion from the border of the wound were visualized and photographed under an inverted microscope (Nikon Ti-u). Migration was quantified by counting the total number of cells that migrated toward the original wound field. A total of three areas were selected randomly from each well and the cells in three wells of each group were quantified in each experiment.

\section{Invasion assay}

Invasion assay was tested on the newer technique of real time migration monitoring using the CIM devices and the XCELLigence DP system (ACEA Biosciences, USA). Before experiment going on, Matrigel was coated on wells respectively. In this system, $8 \times 10^{3}$ treated either with siRNA SLC8A1-AS1 or Noncontrol, then seeded in the upper chamber in the normal culture medium of the respective cell line without FBS. This upper chamber was then placed on the lower part of the CIM-device containing growth medium supplemented with $10 \%$ FBS as an attractant. Invasion of the cells was followed over a time period of up to $120 \mathrm{~h}$ by changes of the impedance signal in a CIM-plate (ACEA Bio) measured on the backside of the membrane and cell growth was monitored in a 16-well e-plate (ACEA Bio) as described for the $x$ CELLigence DP system. Invasion assay was also tested by using Tumor Invasion System (BD BioCoat, BD, NJ) in matrigel coated 24-well inserts. A sample of $3 \times 10^{4}$ cells treated with Noncontrol or SLC8A1-AS1 siRNAs was placed on this system in DMEM-medium without FCS. The inserts were set into DMEM-medium with $10 \%$ FCS as an attractant. After $48 \mathrm{~h}$, the cells were stained with $0.1 \%$ crystal violet for 30 min and observed under a light microscope.

\section{Migration assay}


Cell migration assay were performed by using the newer technique of real time migration monitoring using the CIM devices and the xCELLigence DP system (ACEA Bio). In this system, $8 \times 10^{3}$ cells treated either with siRNA SLC8A1-AS1 or Noncontrol, then seeded in the upper chamber in the normal culture medium of the respective cell line without FBS. This upper chamber was then placed on the lower part of the CIM-device containing growth medium supplemented with $10 \%$ FBS as an attractant. Migration of the cells was followed over a time period of up to $120 \mathrm{~h}$ by changes of the impedance signal in a CIM-plate (ACEA Bio) measured on the backside of the membrane and cell growth was monitored in a 16-well eplate (ACEA Bio) as described for the XCELLigence DP system. Cell migration assay was also tested by using Transwell insert chambers ( $8 \mu \mathrm{m}$ pore size, Corning, USA). A total of $3 \times 10^{4}$ concentration cells were seeded into the upper chambers in serum-free medium. The lower chamber of the Transwell was filled with $500 \mu$ l culture media containing $10 \%$ FBS as a chemo-attractant. After the chambers were incubated at $37^{\circ} \mathrm{C}$ for $48 \mathrm{~h}$, non-invaded cells on the top of the Transwell were scraped off with a cotton swab.

Successfully migrated cells were fixed with $10 \%$ formalin. Then, they were stained with $0.1 \%$ crystal violet for 30 min and counted under a light microscope.

\section{Western blot analysis}

Cell lysates were prepared using sample buffer, laemmli (Sigma, USA) for protein extraction. Protein lysates were separated by $10 \%$ SDS-PAGE, transferred to $0.22-\mu \mathrm{m}$ NC membranes (Milipore, USA) and incubated with antibody. $\beta$-actin was used as a control. Antibodies (1:1000) against claudin, vimentin, E-

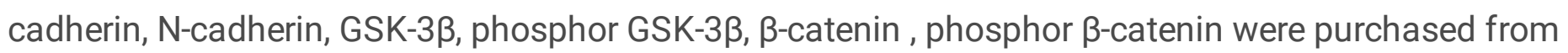
Abcam. $\beta$-actin (1:1000) were purchashed from Sigma. The band intensity was measured by densitometry using the Image $J$. The protein levels were normalized with that of $\beta$-actin. All experiments were repeated in triplicate, and the representative results were shown.

\section{Statistical analysis}

The SPSS 16.0 statistical analysis software was used for the statistical analysis of the experimental data. The significance of differences between groups was estimated by Student's t-test. A p value less than 0.05 were considered significant.

\section{Results}

\section{LncRNA SLC8A1-AS1 is up-regulated in glioma.}

The expression levels of IncRNAs in human 156 glioma tissues and 5 adjacent normal tissues downloaded from The Cancer Genome Atlas (TCGA, https://tcga-data.nci.nih.gov/tcga/) was profiled to explore the functions of IncRNAs in glioma. The results showed that IncRNA SLC8A1-AS1 expression level is significantly up-regulated in glioma tissues $(P \otimes 0.01)$ compared with noncancerous tissues (Figure 1A). We further used real-time RT-PCR to verify the IncRNA SLC8A1-AS1 expression in 67 glioma samples and 7 histologically normal tissues. As shown in Figure 1B, the IncRNA SLC8A1-AS1 expression was significantly over-expressed in glioma tissues (P凶0.001). In addition, receiver operating characteristic 
(ROC) curve analysis was determined to evaluate the diagnostic value of IncRNA SLC8A1-AS1 for glioma. Notably, IncRNA SLC8A1-AS1 displayed the highest accuracy for predicting glioma, because the area under the curve (AUC) was 0.891 in TCGA data $(P=0.0029$, Figure $1 C)$ and 0.99 in our validation queue (P凶0.0001, Figure 1D).

\section{Knockdown of IncRNA SLC8A1-AS1 inhibited glioma cell proliferation and clone formation by inducing cell-cycle arrest in vitro.}

To assess the roles of IncRNA SLC8A1-AS1 in glioma, we down-regulated expression of IncRNA SLC8A1AS1 in human glioma cell lines U87MG and LN382 by transfection with siRNAs. Real time cellular analysis (RTCA) and colony-formation assay were performed to investigate the effects of IncRNA SLC8A1-AS1 on the glioma cells proliferation. The results revealed that down-regulation of IncRNA SLC8A1-AS1 markedly inhibited U87MG and LN382 cells growth (Figure 2A) and decreased the number of colonies formed (Figure 2B). Dysregulation of cell cycle is a vital reason for tumor cell proliferation, to further explore whether IncRNA SLC8A1-AS1 promoted proliferation by infulence cell cycle progression in glioma, we examined cell cycle kinetics by using flow cytometric analysis. The results revealed that U87MG and LN382 cells with siRNAs had an obvious cell cycle arrest in the G2 phase and the population of cells in the $S$ phase was decreased, whereas the G2/M phase fractions increased significantly (Figure 2C). These results confirmed that IncRNA SLC8A1-AS1 is involved in glioma cells progression by inducing cell-cycle arrest.

\section{LncRNA SLC8A1-AS1 regulated glioma cell invasion and migration in vitro.}

We further assessed the effects of IncRNA SLC8A1-AS1 on the invasion and migration capacity of U87MG and LN382 cells. The transwell assay (with or without matrigel coated in the upper chambers) demonstrated that invasion and migration ability of U87MG and LN382 cells was notably decreased with IncRNA SLC8A1-AS1 knockdown (Figure 3A). The wound-healing assay also authenticated this observation and revealed that wounds scraped in confluent cultures of SLC8A1-AS1 siRNA transfected cells closed only partially, whereas nearly full wound closure was observed in the control cells. As shown in Figure 3B, transfection of IncRNA SLC8A1-AS1 siRNA obviously inhibited the time-dependent migration profile of U87MG and LN382 cells. The epithelial mesenchymal transition (EMT) pathway play crucial role in migration and invasion of tumor cells [8,9]. Consequently, we determined the effects of IncRNA SLC8A1-AS1 silencing on EMT pathway. As shown in Figure 3C, down-regulation of IncRNA SLC8A1-AS1 with siRNA treatment increased the protein expression of E-cadherin and Claudin (markers of epithelial phenotype) and decreased expression of $\mathrm{N}$-cadherin and Vimentin (markers of mesenchymal phenotype) in U87MG and LN382 cells. These results confirmed that SLC8A1-AS1 plays a crucial role in glioma cells progression by regulating EMT.

\section{Down-regulation of IncRNA SLC8A1-AS1 suppressed glioma cell Wnt/ $\beta$-catenin pathway.}

To determine the possible mechanism by which SLC8A1-AS1 regulated the migration and invasion of glioma cells, Western-blot analysis was performed to investigate the effects of knockdown of SLC8A1- 
AS1 on the Wnt/ $\beta$-catenin pathway, which is often aberrantly activated in human cancers and contributes to enhanced cell invasion and migration. Western-blot analysis showed that down-regulation of IncRNA SLC8A1-AS1 significantly reduced the levels of phosphorylated GSK-3 $\beta$ (p-GSK3 $\beta$ ) and phosphorylated $\beta$ catenin $\rrbracket p-\beta$-catenin $\rrbracket$, while no detectable changes were observed in the total levels of GSK-3 $\beta$ and $\beta$ catenin. Furthermore, the Hippo pathway are known to play a significant role in glioma proliferation, motility and invasiveness. Our study corroborate that the Hippo pathway doesn't participate in the glioma development $\mathrm{X}$ Figure 4AX.Moreover, inhibition the Wnt/ $\beta$-catenin signaling pathway with XAV939 partly impaired SLC8A1-AS1-induced invasion and metastasis of glioma cells $₫$ Figure 4B $₫$. Taken together, these data suggest that IncRNA SLC8A1-AS1 facilitate the progression of glioma by activating the Wnt/ $\beta$ catenin signaling pathway.

\section{Silencing of IncRNA SLC8A1-AS1 repressed glioma growth in vivo.}

Finally, we validated the effects of IncRNA SLC8A1-AS1 using a nude mouse model with glioma xenografts in vivo. Figure 5A displayed the morphology of tumor xenografts after injection of U87MG cells into the right axilla of nude mice at 35 days. Figure 5B and $\mathrm{C}$ showed the tumor size and average tumor weight in the IncRNA SLC8A1-AS1 silencing group was obviously decreased compared with the control group.

\section{Discussion}

Glioma is the most common primary brain tumors of adults, and represents one of the most aggressive and lethal human cancer types [1]. At present, the prognosis of glioma patients is very poor and the 5-year survival rate less than $10 \%$ [10], even when multimodal treatment strategies are used. Hence, it is an urgent need to clarify the molecular mechanisms underlying progression of glioma and provide evidence for novel therapeutic targets. Many important efforts have been made to identify prognostic molecular biomarkers that could provide explanations regarding glioma formation and progression [11]. Studies have shown that IncRNAs are of great importance in the diagnosis and treatment of tumors. IncRNAPLACT1, which was significantly upregulated in tumor tissues and correlated with progression and poor survival in pancreatic cancer patients [12]. IncRNA NEAT1 contributes to glioblastoma progression through the Wnt/ $\beta$-catenin pathway by scaffolding EZH2 [13]. In the present study, we first analyze the data from the TCGA database and found a novel IncRNA SLC8A1-AS1 was associated with glioma.

LnCRNA SLC8A1-AS1 is oriented in an antisense direction to the protein-coding gene SLC8A1, which is located on chromosome 2 of the human genome [14]. SLC8 gene is a member of $\mathrm{CaCA}\left(\mathrm{Ca}^{2+} / \mathrm{Cation}\right.$ Antiporter) superfamily, encoding $\mathrm{Na}^{+} / \mathrm{Ca}^{2+}$ exchangers (NCX), mainly expressed on cell membrane, mediate $\mathrm{Na}^{+} / \mathrm{Ca}^{2+}$ fluxes across the cell-membrane. Three mammalian genes (SLC8A1, SLC8A2, and $S L C 8 A 3)$ and their splice variants are expressed in different tissues, they all significantly contribute to regulation of $\mathrm{Ca}^{2+}$-dependent events in many cell types [15]. We also observed a marked up-regulation of IncRNA SLC8A1-AS1 in clinical glioma samples detected by real-time RT-PCR. ROC curves were used to evaluate the sensitivity and specificity of IncRNA SLC8A1-AS1 expression in predicting glioma tissues 
from normal tissues, and the results displayed the highest accuracy for predicting glioma. Our results implied that IncRNA SLC8A1-AS1 might act as "oncogene" to promote the progression of glioma and might provide imperative clinical significance in glioma diagnosis.

We then investigated the effects of aberrant IncRNA SLC8A1-AS1 expression on the cellular biological behavior of glioma cells. Abnormal cell proliferation and growth are characteristics of human gliomas [16]. Importantly, IncRNA SLC8A1-AS1 silencing obviously inhibited the proliferation, clone formation, by inducing cell cycle arrest. The results show that knockdown of IncRNA SLC8A1-AS1 leads to reduced cell proliferation in vitro, and in vivo data suggest that it is a potential molecular target for therapy. Cell migration and invasion are significant aspects of cancer progression [17], we found that inhibit the expression of IncRNA SLC8A1-AS1 can inhibited the migration and invasion of U87MG and LN382 cells significantly. EMT is a critical biological process in tumor cell migration and invasion [18]. We found that IncRNA SLC8A1-AS1 siRNA treatment could increase the expression of E-cadherin and claudin, however, decrease the expression of N-cadherin and vimentin at the protein level in U87MG and LN382 cells. LncRNA SLC8A1-AS1 silencing appeared to specifically suppress the progression of EMT in glioma cells, which reduced their invasion abilities. These results indicated that IncRNA SLC8A1-AS1 may serve as a crucial regulator of invasion and migration by inducing EMT in gliomas.

To elucidate the possible mechanism by which IncRNA SLC8A1-AS1 regulates the proliferation, clone formation, migration and invasion of glioma cell, Western-blot analysis of the key molecular factors of cancer-related pathways, such as Hippo pathway, Wnt/ $\beta$-catenin pathway was performed. The Wnt/ $\beta$ catenin pathway plays a key role in tumor EMT[19-21]. GSK-3 $\beta$ is one of the components of the destruction complex in the Wnt/ $\beta$-catenin signaling [22]. In this study, down-regulation of IncRNA SLC8A1AS1 significantly reduced the levels of $p-G S K-3 \beta$ and $p-\beta$-catenin in U87MG and LN382 cells, while no detectable changes were observed in the total levels of GSK-3 $\beta$ and $\beta$-catenin. However, the expression level of phosphorylated YAP-1 (p-YAP-1) in the signal pathway did not change. Furthermore, Wnt/ $\beta$ catenin signaling pathway inhibitor significantly impaired IncRNA SLC8A1-AS1-induced glioma development. These results suggested that IncRNA SLC8A1-AS1 could facilitate the progression of glioma by regulating the $W n t / \beta$-catenin pathway activity.

Although we analysed only two cell lines (U87MG and LN382) in these studies, our nude mouse tumorigenicity assay results provide powerful evidence; therefore, we speculate that other glioma cell lines will show similar results.

\section{Conclusions}

Taken together, we identified for the first time that IncRNA SLC8A1-AS1 is up-regulated in glioma tissues and cell lines. Our findings demonstrated that knockdown of SLC8A1-AS1 inhibits proliferation, migration and invasion of glioma cells. These effects were dependent on Wnt/ $\beta$ catenin activation status, highlighting the potential of IncRNA SLC8A1-AS1 as a candidate therapeutic target in glioma. 


\section{Abbreviations}

LncRNAs: Long non-coding RNAs; EMT: Epithelial to Mesenchymal Transition; TCGA: The Cancer Genome Atlas; WHO: World Health Organization\ATCC: American Type Culture Collection; DMEM: Dulbecco's-modified Eagle medium®FBS: Fetal bovine serum; PI: Propidium iodide; ROC: Receiver Operating Characteristic; AUC: Area under the curve; RTCA: Real time cellular analysis; p-GSK3 $\beta$ : phosphorylated GSK-3 $\beta$; p- $\beta$-catenin: phosphorylated $\beta$-catenin

\section{Declarations}

\section{Authors' contributions}

Ling He performed the research and wrote the manuscript. Hui Yang collected clinical samples and corresponding clinical data. Xiao-long Zhu performed cell culture and the assessment of cell functions in vitro. Yan Zhang and Kun Lv revised the manuscript. All authors read and approved the final manuscript.

\section{Funding}

This work is supported by grants from key projects of Natural Science in Colleges and universities of Anhui province(KJ2019A0424).

\section{Availability of data and materials}

The dataset supporting the conclusions of this article was retrieved by using the TCGA

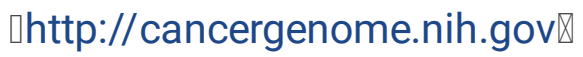

\section{Ethics approval and consent to participate}

All experimental protocols were approved by the Ethics Committee of the The First Affiliated Hospital of Wannan Medical College (Wuhu, China) and performed in accordance with the relevant guidelines and regulations. All animal experiments were approved by the Institutional Animal Care and Use Committee (IACUC) of Wannan Medical College (Wuhu, China)

\section{Acknowledgements}

Not applicable.

\section{Consent for publication}

Not applicable.

\section{Competing interests}

The authors declare no conflict of interest. 


\section{References}

1. Lapointe S, Perry A, Butowski NA. Primary brain tumours in adults. Lancet (London, England). 2018;392(10145):432-46.

2. Laug D, Glasgow SM, Deneen B. A glial blueprint for gliomagenesis. Nature reviews Neuroscience. 2018;19(7):393-403.

3. Delgado-López PD, Corrales-García EM. Survival in glioblastoma: a review on the impact of treatment modalities. Clinical \& translational oncology : official publication of the Federation of Spanish Oncology Societies and of the National Cancer Institute of Mexico. 2016;18(11):1062-71.

4. Ichimura K, Schmidt EE, Goike HM, Collins VP. Human glioblastomas with no alterations of the CDKN2A (p16INK4A, MTS1) and CDK4 genes have frequent mutations of the retinoblastoma gene. Oncogene. 1996;13(5):1065-72.

5. Ichimura K, Schmidt EE, Goike HM, Collins VP. Human glioblastomas with no alterations of the CDKN2A (p16INK4A, MTS1) and CDK4 genes have frequent mutations of the retinoblastoma gene. Oncogene. 1996;13(5):1065-72.

6. Xu H, Zhao G, Zhang Y, Jiang H, Wang W, Zhao D, et al. Long non-coding RNA PAXIP1-AS1 facilitates cell invasion and angiogenesis of glioma by recruiting transcription factor ETS1 to upregulate KIF14 expression. Journal of experimental \& clinical cancer research : CR. 2019;38(1):486.

7. Jiang J, Wang X, Gao G, Liu X, Chang H, Xiong R, et al. Silencing of IncRNA HOXA11-AS inhibits cell migration, invasion, proliferation, and promotes apoptosis in human glioma cells via upregulating microRNA-125a: in vitro and in vivo studies. American journal of translational research. 2019;11(10):6382-92.

8. Huarte M. The emerging role of IncRNAs in cancer. Nature medicine. 2015;21(11):1253-61.

9. Ye X, Brabletz T, Kang Y, Longmore GD, Nieto MA, Stanger BZ, et al. Upholding a role for EMT in breast cancer metastasis. Nature. 2017;547(7661):E1-e3.

10. 10Tang X, Zhao S, Zhang Y, Wang Y, Zhang Z, Yang M, et al. B7-H3 as a Novel CAR-T Therapeutic Target for Glioblastoma. Molecular therapy oncolytics. 2019;14:279-87.

11. 11Xu J, Zhang Z, Qian M, Wang S, Qiu W, Chen Z, et al. Cullin-7 (CUL7) is overexpressed in glioma cells and promotes tumorigenesis via NF-KB activation. Journal of experimental \& clinical cancer research : CR. 2020;39(1):59.

12. 12 Ren $X$, Chen $C$. IncRNA-PLACT1 sustains activation of NF-KB pathway through a positive feedback loop with IKBa/E2F1 axis in pancreatic cancer. 2020;19(1):35.

13. Chen Q, Cai J, Wang Q, Wang Y, Liu M, Yang J, et al. Long Noncoding RNA NEAT1, Regulated by the EGFR Pathway, Contributes to Glioblastoma Progression Through the WNT/ $\beta$-Catenin Pathway by Scaffolding EZH2. Clinical cancer research : an official journal of the American Association for Cancer Research. 2018;24(3):684-95.

14. Guo GL, Sun LQ, Sun MH, Xu HM. LncRNA SLC8A1-AS1 protects against myocardial damage through activation of cGMP-PKG signaling pathway by inhibiting SLC8A1 in mice models of myocardial 
infarction. 2019;234(6):9019-32.

15. Khananshvili D. The SLC8 gene family of sodium-calcium exchangers (NCX) - structure, function, and regulation in health and disease. Molecular aspects of medicine. 2013;34(2-3):220-35.

16. Hanahan D, Weinberg RA. Hallmarks of cancer: the next generation. Cell. 2011;144(5):646-74.

17. Oh JM, Venters CC, Di C, Pinto AM, Wan L, Younis I, et al. U1 snRNP regulates cancer cell migration and invasion in vitro. 2020;11(1):1.

18. Mi L, Zhu F, Yang X, Lu J, Zheng Y, Zhao Q, et al. The metastatic suppressor NDRG1 inhibits EMT, migration and invasion through interaction and promotion of caveolin-1 ubiquitylation in human colorectal cancer cells. Oncogene. 2017;36(30):4323-35.

19. Nusse R, Clevers H. Wnt/ $\beta$-Catenin Signaling, Disease, and Emerging Therapeutic Modalities. Cell. 2017;169(6):985-99.

20. Gonzalez DM, Medici D. Signaling mechanisms of the epithelial-mesenchymal transition. Science signaling. 2014;7(344):re8.

21. Kahlert UD, Maciaczyk D, Doostkam S, Orr BA, Simons B, Bogiel T, et al. Activation of canonical WNT/ $\beta$-catenin signaling enhances in vitro motility of glioblastoma cells by activation of ZEB1 and other activators of epithelial-to-mesenchymal transition. Cancer letters. 2012;325(1):42-53.

22. MacDonald BT, Tamai K, He X. Wnt/beta-catenin signaling: components, mechanisms, and diseases. Developmental cell. 2009;17(1):9-26.

\section{Figures}


A

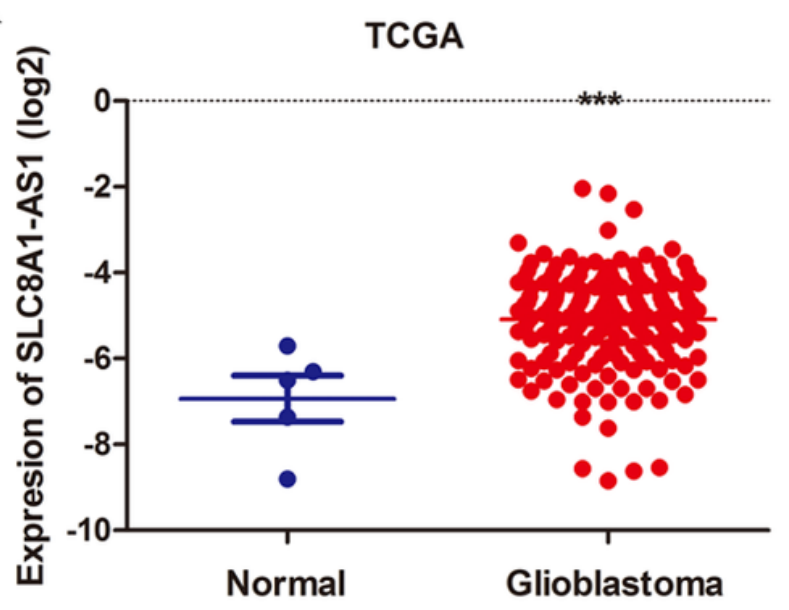

C

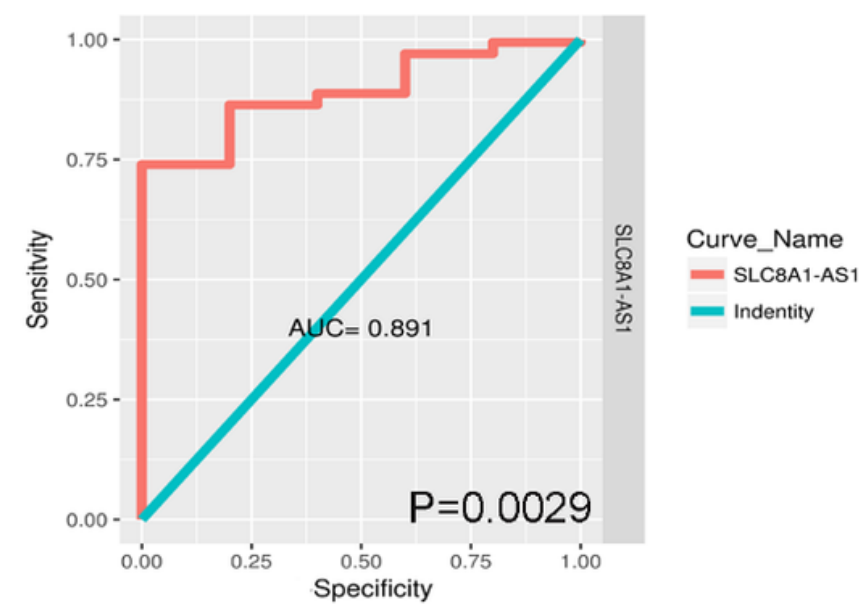

B

Tisstues

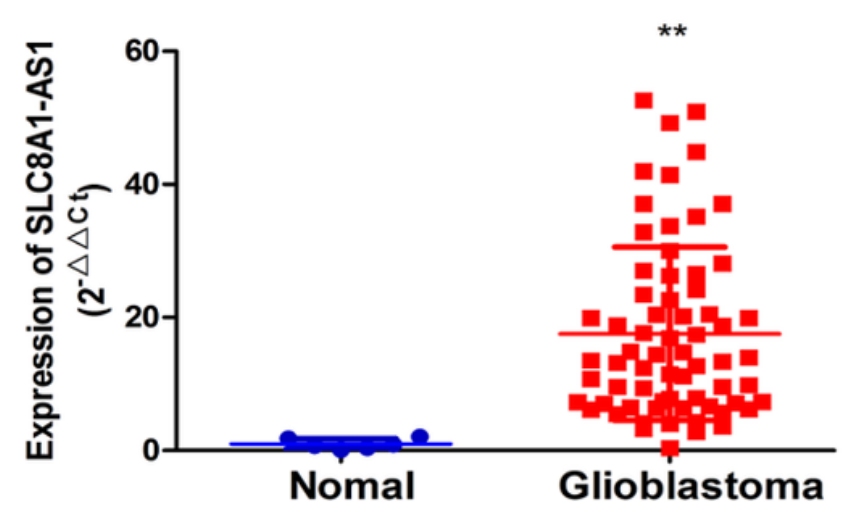

D

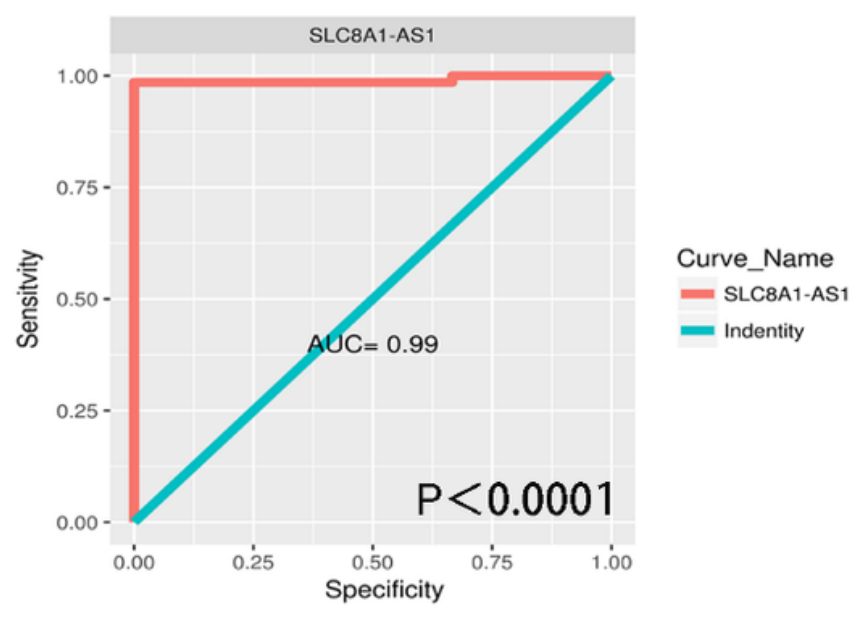

Figure 1

LncRNA SLC8A1-AS1 is up-regulated in glioma. A. Relative expression levels of SLC8A1-AS1 in TCGA database. B. Relative expression of SLC8A1-AS1 in the tumor tissues $(n=67)$ compared to normal tissues $(n=7)$. SLC8A1-AS1 expression was evaluated by qRT-PCR and normalized to GAPDH expression. C.ROC curve for prediction of glioblastoma based on SLC8A1-AS1 expression, using corresponding adjacent normal tissues as a control. D. ROC curve for prediction of glioma based on SLC8A1-AS1 expression, using corresponding normal tissues as a control. ***P $<0.001$ 
A.

U87MG

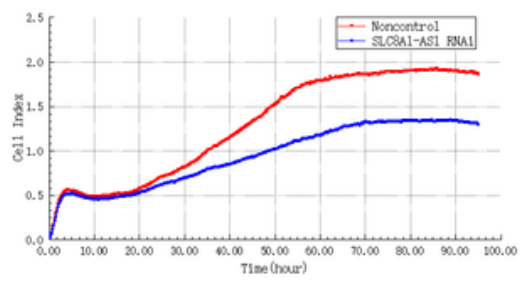

LN382

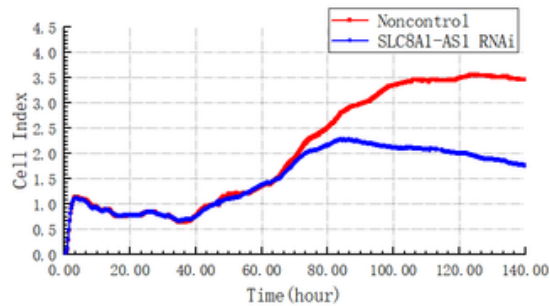

B.
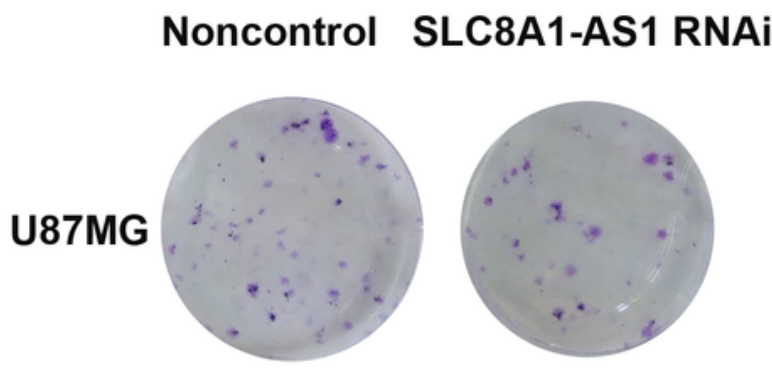

LN382
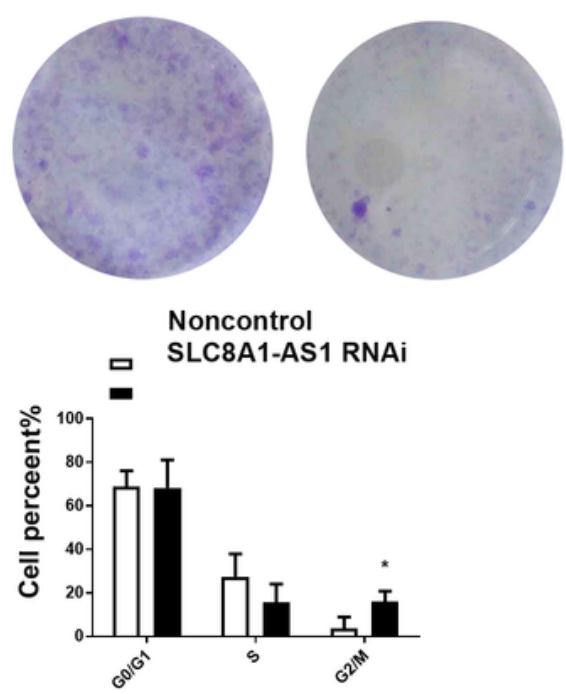
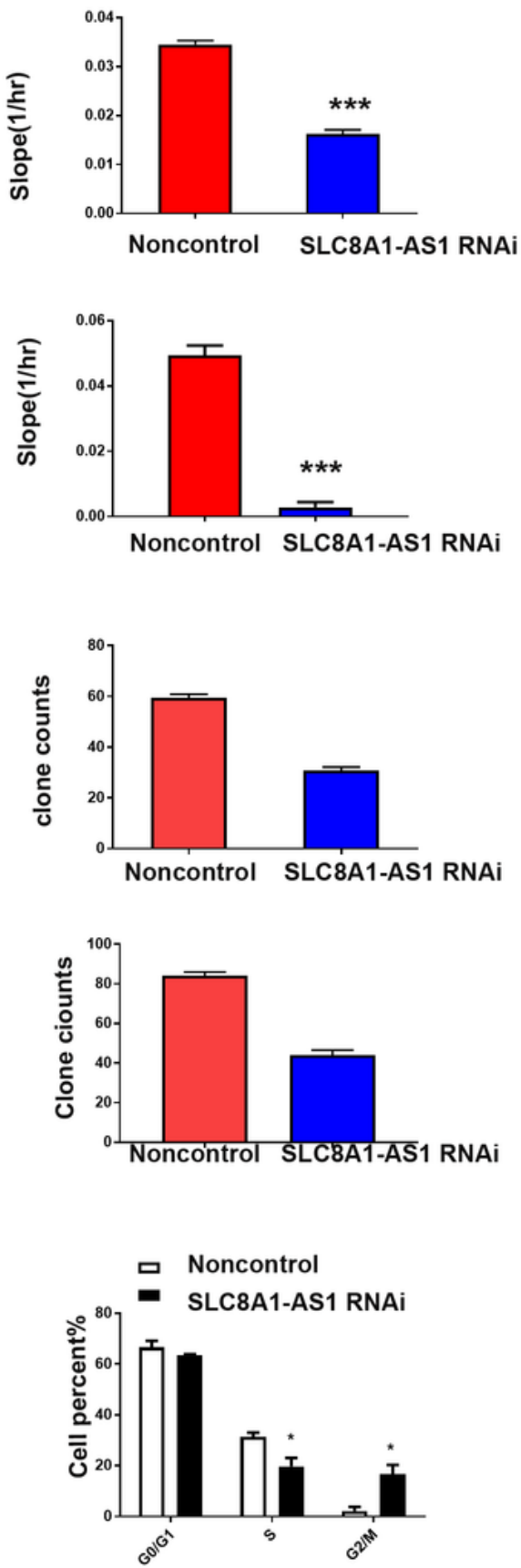

Figure 2

Knockdown of IncRNA SLC8A1-AS1 inhibited glioma cell proliferation in vitro.A. RTCA system were performed to determine the proliferation of SLC8A1-AS1 siRNA transfected U87MG and LN382 cells. B. Colony-forming assays were conducted to determine the proliferation of SLC8A1-AS1 siRNA transfected U87MG and LN382 cells. C. Flow cytometric analysis were conducted to determine the cell cycle of 
SLC8A1-AS1 siRNA transfected U87MG and LN382 cells. The experiments were performed in triplicate,

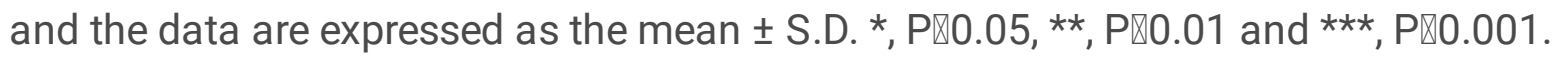

A.
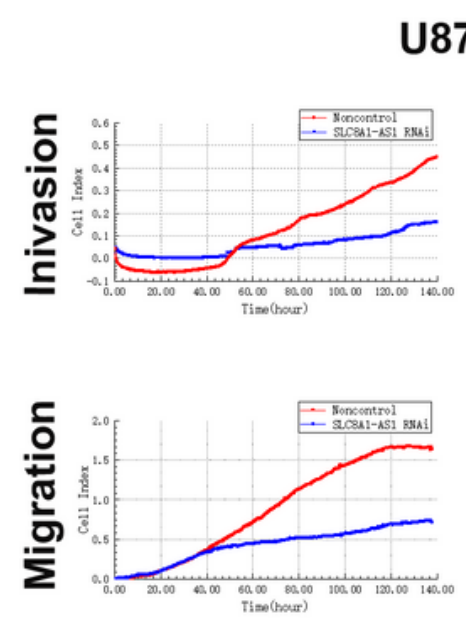

B.
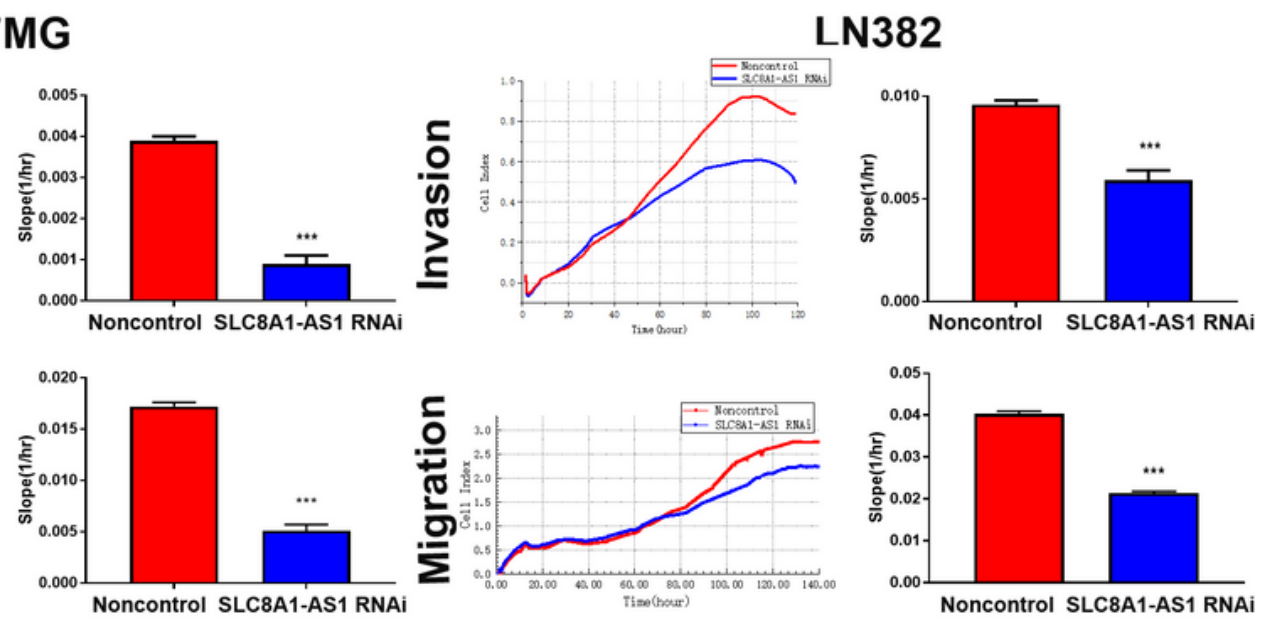

\section{U87MG}

\section{LN382}
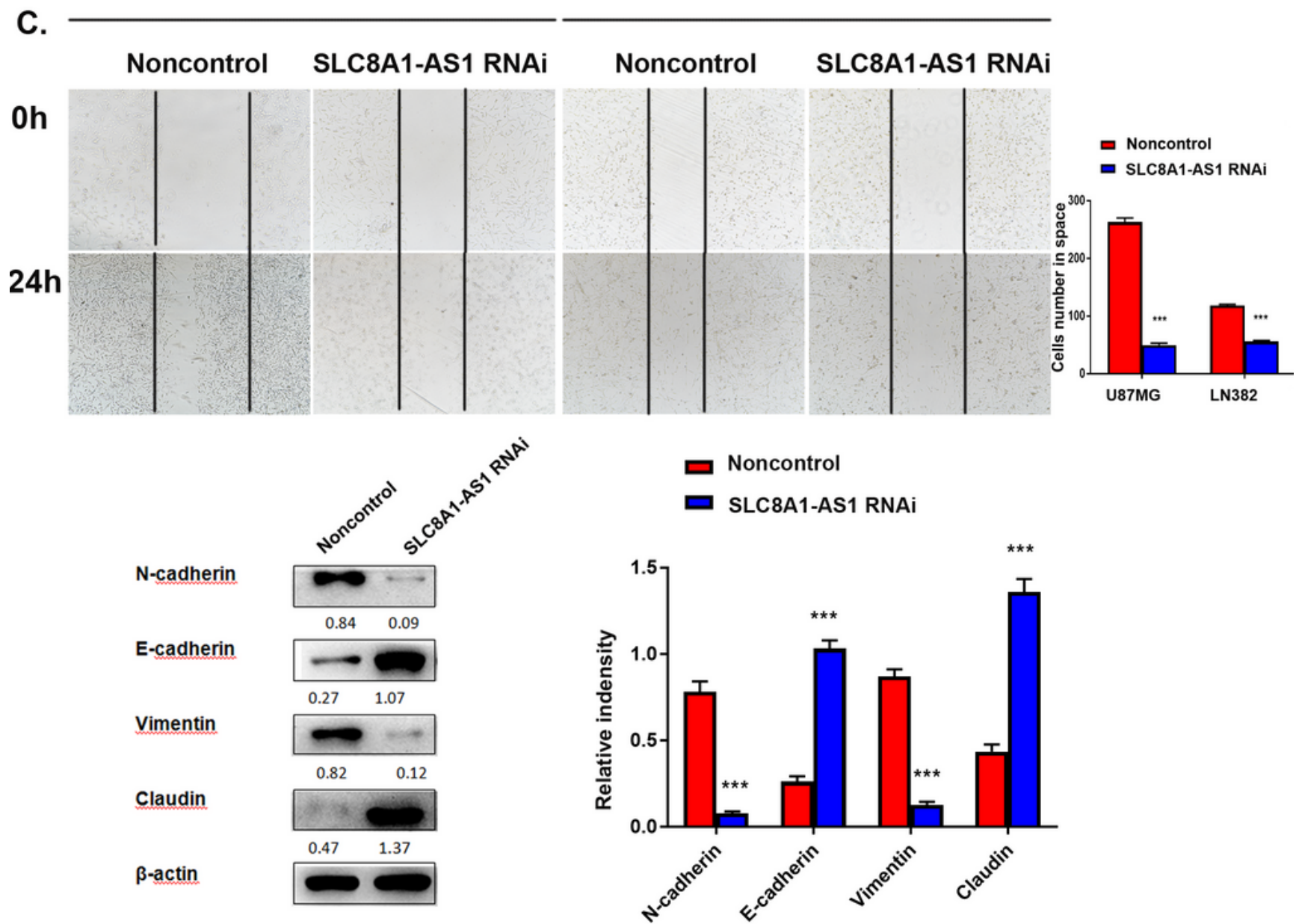

\section{Figure 3}

LncRNA SLC8A1-AS1 regulated glioma cell invasion and migration in vitro. A. The U87MG and LN382 cell invasion was performed by RTCA DP system. B. Wound-healing assays were used to determine the effects of SLC8A1-AS1 knockdown on the migration ability of U87MG and LN382 cells. C. The EMT 
pathway markers was assessed by western-blot. The experiments were performed in triplicate, and the data are expressed as the mean \pm S.D. *, Pख0.05, **, Pख0.01 and ***, Pख0.001.

A.

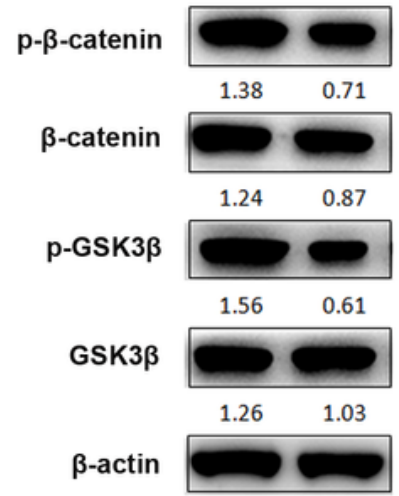

B.

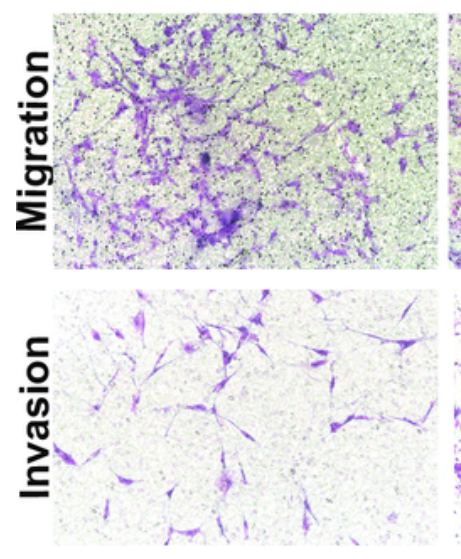

C.
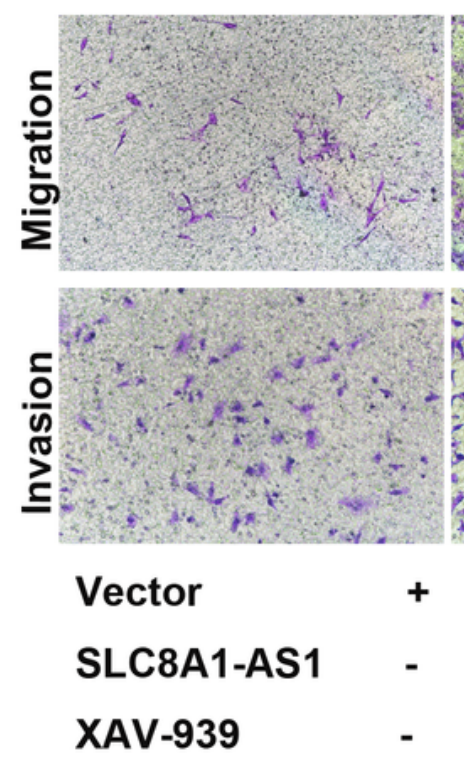

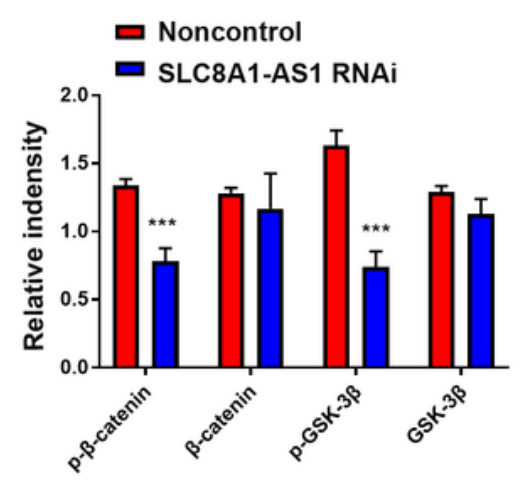

U87MG

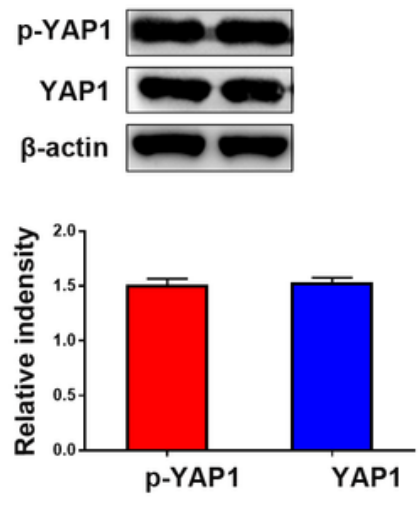

U87MG

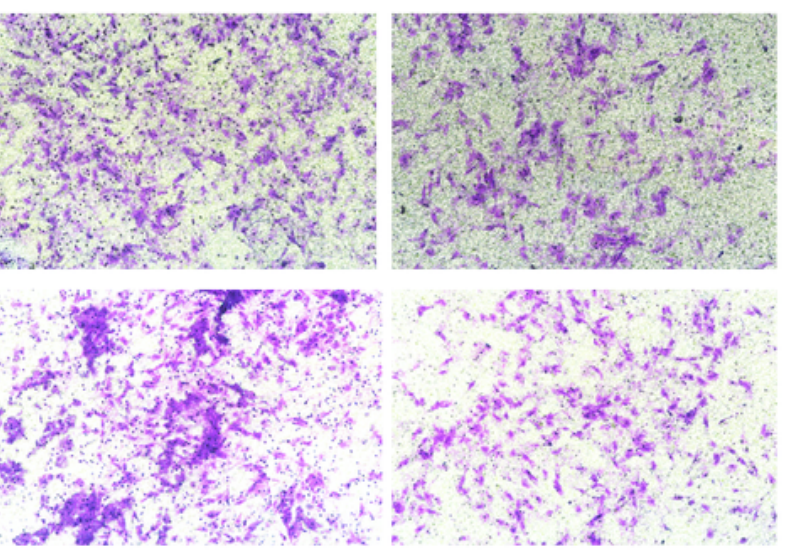

LN382
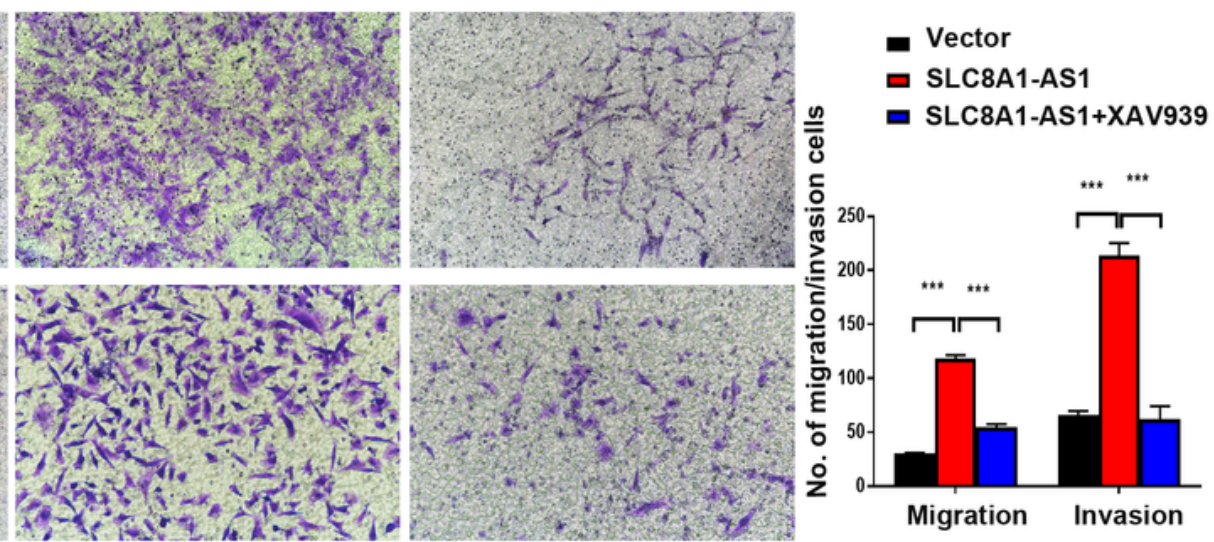

- SLC8A1-AS1+XAV939

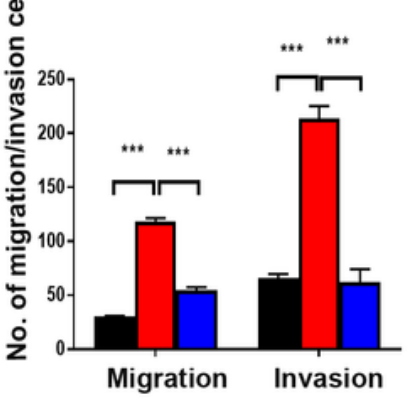

\section{Figure 4}

Down-regulation of IncRNA SLC8A1-AS1 suppressed glioma cell Wnt/ $\beta$-catenin pathway. A. Effect of SLC8A1-AS1 knockdown on Wnt/ $\beta$-catenin and Hippo pathway. B. Representative images of transwell assays showed that XAV939 reversed the effects of SLC8A1-AS1-overexpressing glioma cells. Scale bars: 
$100 \mu \mathrm{m}$. p-values were calculated by using two-tailed t-tests. The error bars represent standard deviations of three independent experiments. ${ }^{*} p<0.05,{ }^{*} \mathrm{p}<0.01, * \star \star, \mathrm{P} \otimes 0.001$.
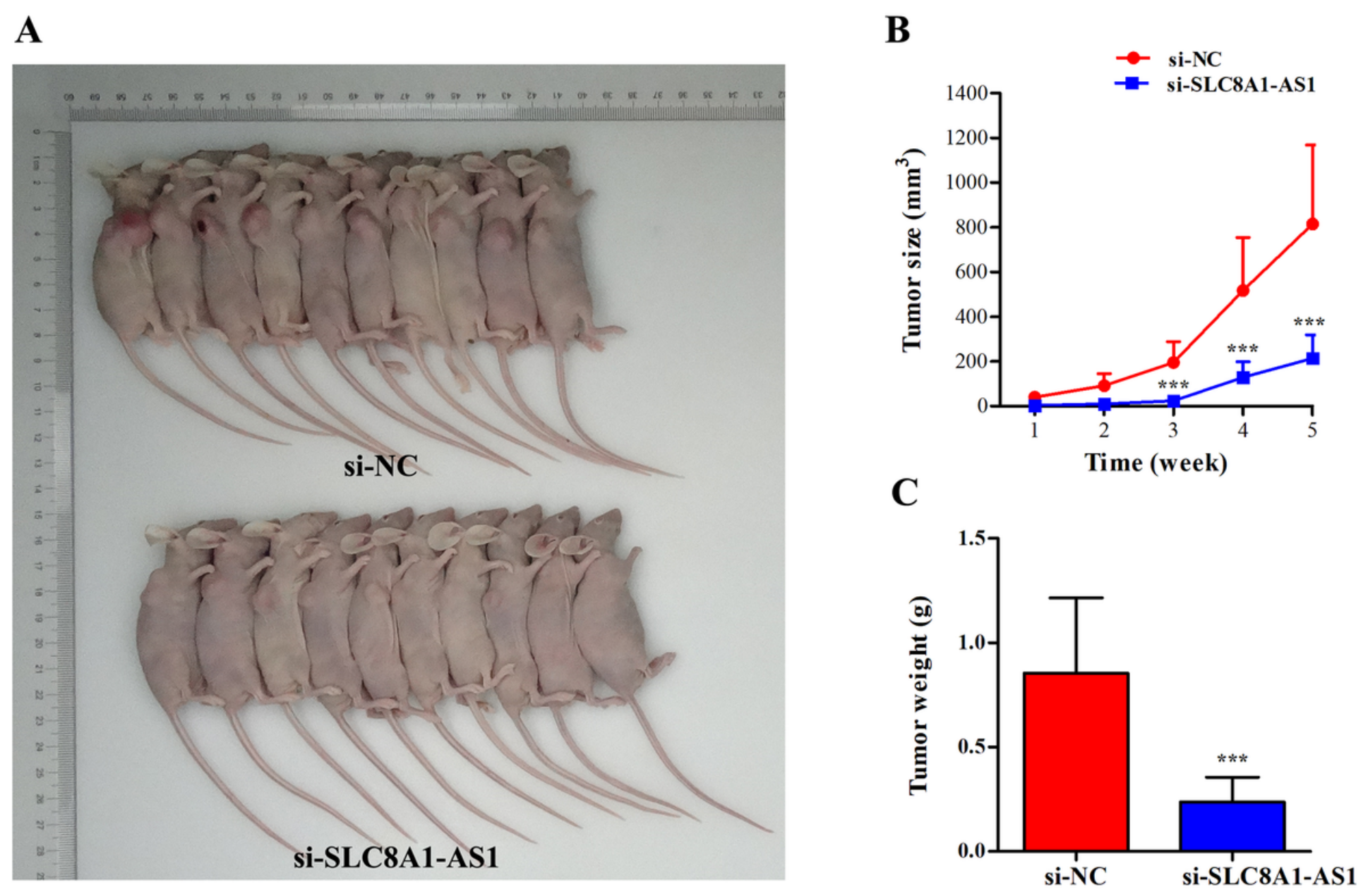

\section{Figure 5}

SLC8A1-AS1 suppressed U87MG and LN382 cell proliferation in vivo. A. U87MG cells with stable SLC8A1AS1 knockdown and control cells were inoculated into nude mice. These graphs show the tumor xenografts 6 weeks after ectopic-subcutaneous implantation in nude mice. B. U87MG cells with downregulated SLC8A1-AS1 expression exhibited attenuated tumor growth in nude mice. The effect of SLC8A1-AS1 on glioma growth was evaluated based on tumor volume in the two groups. 